\title{
Review Article \\ Separation and Characterization of Synthetic Polyelectrolytes and Polysaccharides with Capillary Electrophoresis
}

\author{
Joel J. Thevarajah, ${ }^{1,2}$ Marianne Gaborieau, ${ }^{1,2}$ and Patrice Castignolles ${ }^{1}$ \\ ${ }^{1}$ University of Western Sydney (UWS), School of Science and Health, Australian Centre for Research on Separation Sciences (ACROSS), \\ Parramatta, NSW 2751, Australia \\ ${ }^{2}$ University of Western Sydney (UWS), School of Science and Health, Molecular Medicine Research Group (MMRG), \\ Parramatta, NSW 2751, Australia
}

Correspondence should be addressed to Patrice Castignolles; p.castignolles@uws.edu.au

Received 30 April 2014; Revised 31 July 2014; Accepted 31 July 2014; Published 14 September 2014

Academic Editor: Alejandro Sosnik

Copyright (C) 2014 Joel J. Thevarajah et al. This is an open access article distributed under the Creative Commons Attribution License, which permits unrestricted use, distribution, and reproduction in any medium, provided the original work is properly cited.

\begin{abstract}
The development of macromolecular engineering and the need for renewable and sustainable polymer sources make polymeric materials progressively more sophisticated but also increasingly complex to characterize. Size-exclusion chromatography (SEC or GPC) has a monopoly in the separation and characterization of polymers, but it faces a number of proven, though regularly ignored, limitations for the characterization of a number of complex samples such as polyelectrolytes and polysaccharides. Free solution capillary electrophoresis (CE), or capillary zone electrophoresis, allows usually more robust separations than SEC due to the absence of a stationary phase. It is, for example, not necessary to filter the samples for analysis with CE. CE is mostly limited to polymers that are charged or can be charged, but in the case of polyelectrolytes it has similarities with liquid chromatography in the critical conditions: it does not separate a charged homopolymer by molar mass. It can thus characterize the topology of a branched polymer, such as poly(acrylic acid), or the purity or composition of copolymers, either natural ones such as pectin, chitosan, and gellan gum or synthetic ones.
\end{abstract}

\section{Introduction to CE and Limitations of Size- Exclusion Chromatography (SEC/GPC)}

Free solution capillary electrophoresis (CE), or capillary zone electrophoresis, is a robust polymer separation method. CE differs from the commonly known slab electrophoresis or capillary gel electrophoresis as the capillary does not contain any stationary phase: it is just filled with a buffer (also named background electrolyte). CE does not require tedious sample preparation, not even filtration (e.g., see later in Section 3.2.2). It has several advantages over traditional separation techniques for the characterization of polyelectrolytes which will be outlined in this review. The most commonly used method for the separation and characterization of polymers is size-exclusion chromatography (SEC, also known as GPC). SEC is relatively quick and affordable in obtaining data regarding the size or molar mass of a polymer with good repeatability [1]. Among
SEC's main limitations is its poor reproducibility in terms of molar mass analysis: round-robin tests often show poor accuracy of the values of the determined molar mass [2]. This is detailed in Berek's recent critical review [3]. The review linked the common accuracy issue to the difficulties in obtaining a pure size-exclusion separation: secondary retention mechanisms, side processes, parasitic processes, osmotic effects, secondary exclusion, concentration effects, preferential interactions, and SEC band broadening. For the ultrahigh molar masses, the sample is generally thought to be degraded by shear [4], although a change of conformation of the polymer chains may also take place, leading to a new separation mechanism [5]. In addition, even in ideal conditions (pure size-exclusion mechanism, no degradation), SEC separates by hydrodynamic volume not by molar mass [6]. Apparent molar masses determined by SEC (e.g., polystyrene-equivalent molar masses) thus have a variable and sometimes limited accuracy $[7,8]$. Different topologies 
(branching) or compositions of the polymer sample influence the hydrodynamic volume and the separation is then incomplete in terms of molar mass when a range of branching structures or of compositions is present in a sample [9-11]. This can render the simple determination of molar mass using Mark-Houwink-Sakurada parameters inaccurate, like in the case of most poly(alkyl acrylates) $[12,13]$. Up to $100 \%$ error in the determination of the molar mass of branched polymers has been measured using multiple detection SEC (light scattering and viscometry) [14, 15].

We recently discussed the SEC of branched polymers and polysaccharides in a review [16]. Composition of copolymers, branching, and purity are often overlooked in polymer characterization, since SEC has a quasi-monopoly and is not suited for these types of characterization. However, alternative methods are being developed, especially alternative liquid chromatography methods [17]. Liquid chromatography in critical conditions (or at the critical conditions) [18] is one of the most prominent alternative chromatography technique: the critical conditions for one homopolymer correspond to the absence of separation by molar mass for this homopolymer, allowing for separation solely by its topology if branched [19] or solely by its composition if copolymerized [20]. These critical conditions are, however, tedious to establish and low recoveries have been observed [21, 22]. CE offers an alternative and the objective of this review is to present and discuss the potential of $\mathrm{CE}$ for synthetic polymers and polysaccharides.

CE (defined here as free solution capillary electrophoresis) involves separation in a capillary filled with only buffer (no stationary phase) under high voltage [23]. The use of only a buffer and no stationary phase prevents the common problem of adsorption onto the stationary phase (and of degradation or deformation of the ultrahigh molar mass chains) commonly faced in SEC. The velocity of different analytes is proportional to the electric field: the proportional constant is named the electrophoretic mobility, $\mu_{\mathrm{ep}}$. The selectivity of CE separation relates to the difference in electrophoretic mobility of the analytes (see Figure 1 for the experimental determination of $\mu_{\mathrm{ep}}$ ). The electroosmotic flow (EOF) is created by the movement of the ions of the background electrolyte through the capillary under electric field. The EOF is contributing to the migration of all molecules, even neutral ones. At a high $\mathrm{pH}$ the silanol groups of the glass layer of the capillary are completely ionized. This generates a strong zeta potential and an electrical double layer of silanolate groups and positive ions from the background electrolyte. The higher the $\mathrm{pH}$, the higher the density of the electrical double layer which increases the EOF [23].

Successful applications of CE to polymer characterization have been the object of a number of publications, especially by Cottet's group, and the earliest works have been reviewed [24]. Building on these advances, using CE, our group was able to reliably characterize several natural and synthetic polymers, especially polysaccharides and poly(acrylic acid) as discussed in this review.

\section{Free Solution Capillary Electrophoresis (CE)}

Characterization of polymers by $\mathrm{CE}$ can be divided into at least four categories: separation of monomer units after depolymerization (see Section 2.1), separation of oligoelectrolytes (see Section 2.2), and separation of longer polyelectrolytes (see Section 3). The fourth category is the separation of polymers bearing a single charge or no charge. For the latter category, the reader is referred to the pioneering work of the groups of Cottet [25, 26] and Cifuentes [24, 27].

\subsection{Average Composition of Polysaccharides}

2.1.1. Robust Separation of a Mixture of Monosaccharides. A number of polysaccharides, such as hemicellulose [28, 29], have highly complex chemical structures: they are composed of several different monomer units, mainly monosaccharides. The analysis of these polysaccharides is extremely difficult. The average composition can be determined after depolymerization (hydrolysis) and quantification of the different resulting monosaccharides. Currently high performance liquid chromatography (HPLC) is used to separate carbohydrates using different modes; however, this technique and the different modes used have limitations in regard to coelution [30], tedious sample preparation, and short column life [31]. The detection of monosaccharides is another difficulty. CE is most easily and classically applied to analytes that are charged and possess chromophores. The $\mathrm{pKa}$ of most mono- and disaccharides is around $12[32,33]$ and separation in CE was obtained at high $\mathrm{pH}$ [34] but initially indirect UV detection, conductivity detection, or derivatization was required for detection. Rovio et al. showed that different hemicelluloses can be characterized not only with CE but also with direct UV detection [32, 33]. This method was applied to plant fiber samples without any derivatization: $\mathrm{CE}$ achieved a highresolution separation of the depolymerized fiber samples (Figure 1) and was compared to the various common HPLC methods and IC (HPAEC) [30,32]. The CE separation can be optimized at minimal cost by changing the capillary length, buffer counter-ion, and/or the buffer concentration [35]. The main advantage of $\mathrm{CE}$ is the robustness of the technique, especially the minimum sample preparation that is required. The precision of the peak identification and the quantification were greatly improved with the use of an electroosmotic flow (EOF) marker and an internal standard [30]. Figure 1 shows electropherograms when raw data (migration time) are compared to corrected data (electrophoretic mobility). Figure 1 also gives the equation used to perform this transformation. In the equation, $\mu_{\mathrm{ep}}$ is electrophoretic mobility, $V$ is voltage, $L_{d}$ is the length to the detector, $L_{t}$ is the total length of the capillary, $t_{m}$ is the time of migration, and $t_{e o}$ is the migration of a neutral species. Using electrophoretic mobility (thus correcting for EOF variations) allows easy visual comparison of results, for example, to allow identification of trace sugars in ethanol fermentation [36].

$\mathrm{CE}$ was able to resolve and quantify mannose, galactose, and xylose. The CE quantification of these sugars results in larger amounts when compared to the HPLC results. This might indicate incomplete recovery in HPLC possibly due 


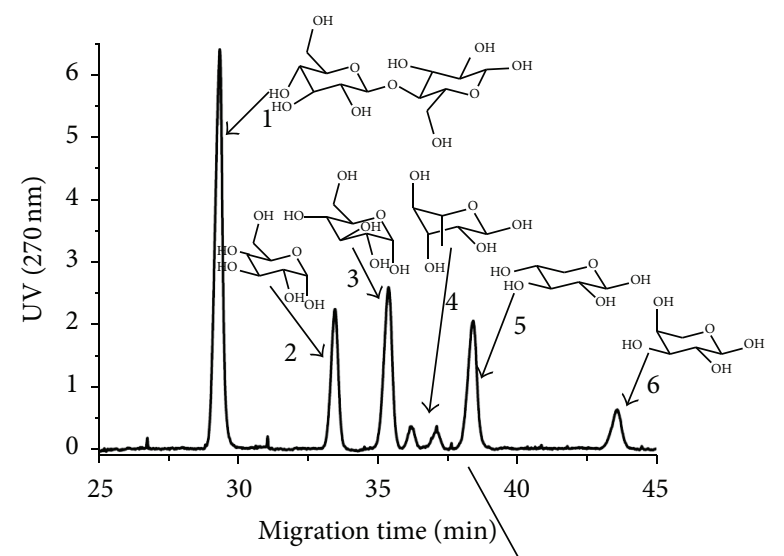

(a)

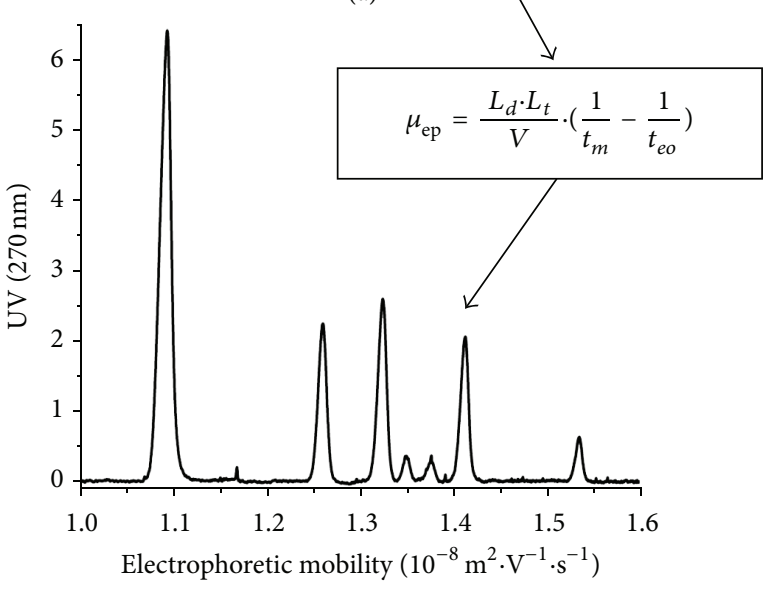

(b)

FIgURE 1: Separation by CE at high pH (12.6) and with direct UV detection of a depolymerized plant fiber sample plotted as a function of electrophoretic mobility (a) and of migration time (b). The sample contains (1) cellobiose, (2) galactose, (3) glucose, (4) rhamnose, (5) arabinose, and (6) xylose (the molecular structures are given for the sole purpose of identification, and they are in equilibrium with a number of linear and charged forms) [30].

to adsorption onto the stationary phase, which is a common problem associated with the HPLC of samples in complex matrices. There were weaknesses in the direct UV detection in CE which have been addressed recently (see Section 2.1.2).

\subsubsection{Direct Detection due to the Photooxidation of Sugars.} Rovio et al. [33] showed that the detection of monosaccharides was possible at $270 \mathrm{~nm}$ at $\mathrm{pH}$ 12.6. Sarazin et al. [43] suggested the method of detection was due to a photooxidation reaction occurring at the detection window. We confirmed that the detection is by photooxidation using a combination of simulation, multidimensional CE migration, and NMR spectroscopy analysis $[30,37]$. The detection occurs without the electric field (i.e., in pressure mobilization instead of CE) but the electric field enhances the sensitivity of the detection. The photooxidation is initiated either by hydroxyl radicals formed by minimal but sufficient water decomposition or by direct decomposition of the carbohydrates under UV irradiation [35]. The diode-array detector (DAD) emits UV light down to $190 \mathrm{~nm}$. These wavelengths are not leading to any known sample degradation, except for the photooxidation of carbohydrates at high $\mathrm{pH}$. The photooxidation is a type of in situ derivatization. If one wishes to avoid the photooxidation reaction taking place, then the lowest wavelengths need to be filtered out: on commercial equipment this simply means using UV detection and not a DAD. Even by using a $\mathrm{DAD}$, most of the sugar molecules are not photooxidized (in the timeframe of the detection). The UV-absorbing species are intermediates in the photooxidation process [37]. These intermediates are present at low concentration but have a high UV absorption coefficient. The final products do not absorb UV and are likely obtained after reacting with oxygen. NMR spectroscopy used as offline detection after $\mathrm{CE}$ migration allowed for the identification of a number of carboxylated compounds in the final products. This CE method is ideally suited for the separation of mono- and disaccharides in complex matrices. Direct detection has the advantage of simplicity and of using the most common detector in CE (diode-array detection). The detection of the CE was found to have a limit of detection 10-100x better than HPLC and a better selectivity of detection. Direct detection in $\mathrm{CE}$ is not as sensitive as more convoluted methods based on derivatization or pulsed-amperometric detection in ionchromatography [36]. The method will thus need further improvement to be used for trace detection. Preliminary results showed that using a radical photoinitiator can increase the sensitivity of the direct UV detection [37].

The CE method (Figure 2) is useful not only to determine the average composition of complex polysaccharides, but also to monitor carbohydrates, for example, in a fermentation process. The latest developments showed that fermentation products such as ethanol can also be determined [35]. Ethanol is inhibiting the photooxidation process and this leads to indirect detection of ethanol in the presence of a sugar, such as sucrose. This indirect detection was successfully applied to monitoring lignocellulosic fiber fermentation in terms of both ethanol and sugars alcohols [36].

2.2. Oligoelectrolytes. Cottet and Gareil have shown that oligo(styrene sulfonate)s can be separated by their molar mass up to a degree of polymerization of 9 [44]. Oligo(sodium acrylates)_oligoAAs_-are used in the paint and coating industries to stabilize emulsions [45]. Controlled polymerization methods such as reversible addition-fragmentation chain transfer (RAFT) allow the controlled synthesis of oligoAA. CE can separate oligoAAs (Figure 3) at a higher resolution than that ever obtained with SEC (for oligomers) [38] even using optimal SEC conditions [46, 47].

$\mathrm{CE}$ was able to separate and quantify the residual RAFT agent used to obtain the oligoAAs as well as the species of degrees of polymerization (DP) of one, two, and three. The identification of these peaks was obtained by the online coupling of CE with ESI-MS-TOF (electrospray ionizationmass spectrometry-time of flight) [48]. MS analysis showed that the oligomers are separated not only according to their 


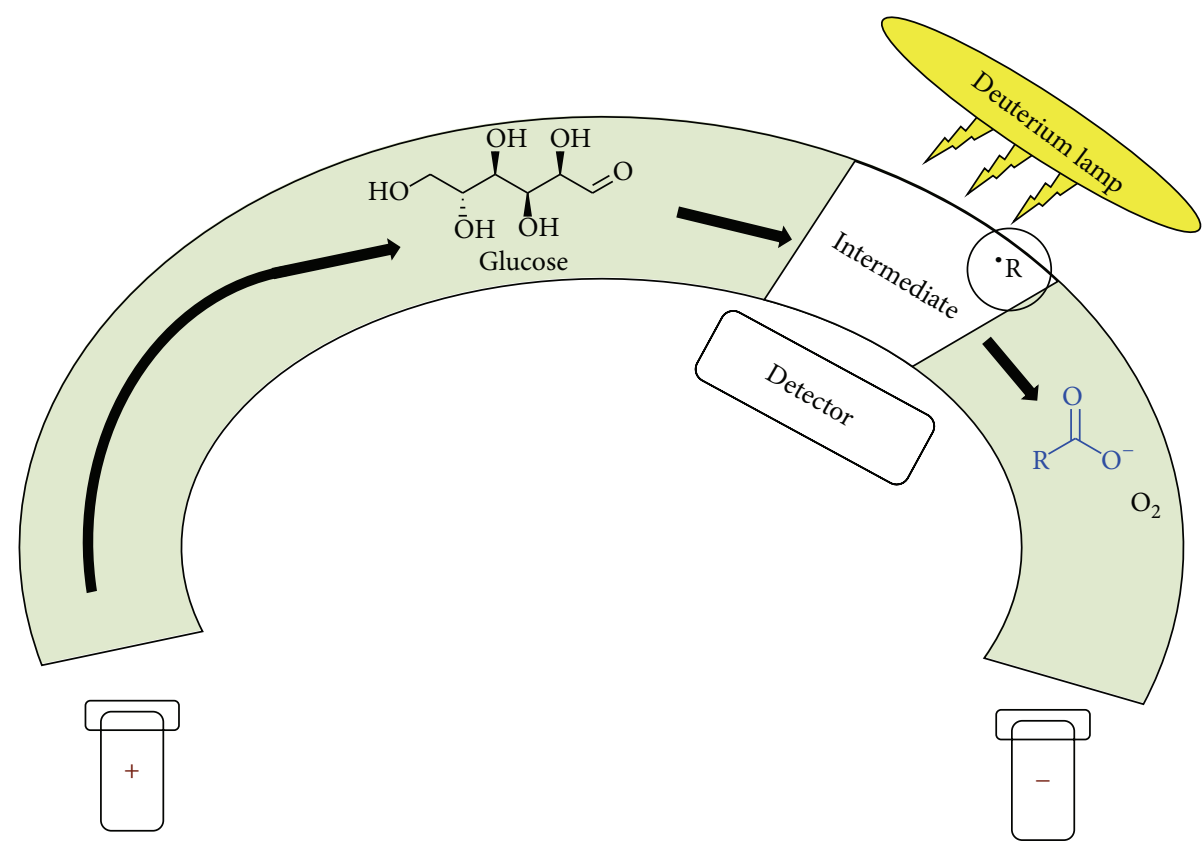

FIGURE 2: Mechanism of direct UV detection in CE of carbohydrates owing to a photooxidation reaction [37].

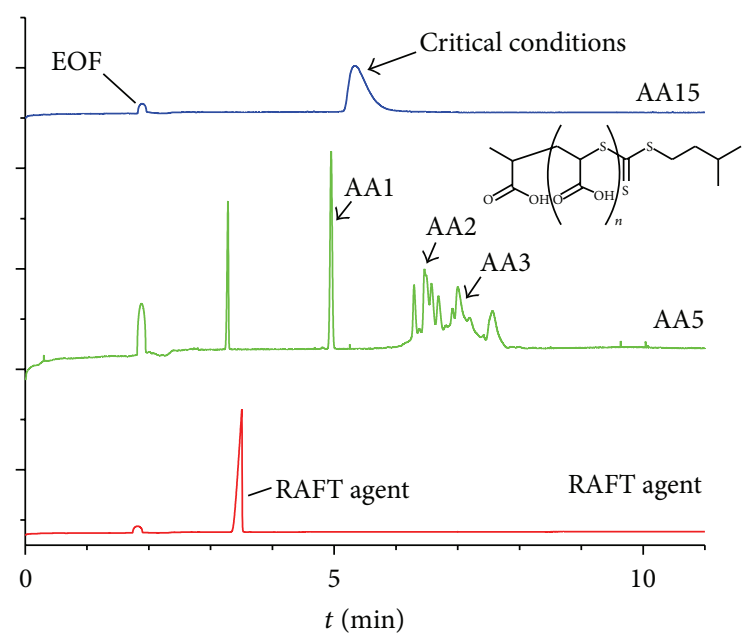

FIGURE 3: Electropherograms in lithium borate for two different oligoAAs, AA5 and AA15, produced by RAFT polymerization, where 5 and 15 correspond to the degree of polymerization obtained at the maximum of the mass spectrum from ESI-MS-TOF (electrospray ionization-mass spectrometry-time of flight) direct infusion (adapted from [38]). AA15 is not separated by molar mass; it is thus in the critical conditions. The bottom electropherogram is of the RAFT agent, that is, the control agent for the polymerization.

degree of polymerization and end-group, but also according to their tacticity. The shortest oligoAAs were shown to contain $50 \%$ of unreacted RAFT agent, while the direct infusion in ESI-MS estimated that the sample contained only $2 \%$ of unreacted RAFT agent. This large discrepancy is due to the known issue of the bias of the ionization towards low degrees of polymerization and hydrophilic species in MS analysis [49]. CE was shown to be a relevant and fast method in the study of kinetics of polymerization of RAFT. It has also been used to shed light on the kinetics and mechanism of ring opening polymerization of either 2-oxazoline [50] or $N$ carboxyanhydrides [26].

Most importantly, the high-resolution separation of CE by molar mass is limited to oligoelectrolytes, with degrees of polymerization below about 10. For large polyelectrolytes, no separation by molar mass is obtained, which corresponds to the "critical conditions" described below.

\section{3. $\mathrm{CE}$ in the Critical Conditions}

3.1. Explanation of "Critical Conditions". The first example of analysis of synthetic polyelectrolytes by electrophoresis dealt with poly (4-vinyl- $N$ - $n$-butylpyridinium bromide) more than half a century ago [51]. The authors concluded that "the electrophoretic behavior of polyvinylbutylpyridinium is not very sensitive to molecular weight." CE in the "critical conditions" differs from the CE undertaken in the separation and characterization of oligoelectrolytes such as oligoAA. Critical conditions refer to the conditions sought in liquid chromatography (LC) in which a homopolymer is not separated by molar mass (see Section 1). While these critical conditions are of no use to characterize simple (homo)polymers, most, if not all, polymers are not simple in the sense that they possess a distribution of molar masses as well as different end-groups, distribution(s) of compositions for copolymers, distribution of branch molar masses and of positions of branching points for branched polymers, and so forth. Polymeric samples are multidimensional: the critical conditions allow separation by one (or only a few) dimension at one time (since the distribution of molar masses does not influence the separation any more). The critical conditions thus enable the characterization of complex polymers through the 
simplification of a multidimensional problem. While a lot of research has been devoted to LC and critical conditions, the method remains tedious and plagued with low accuracy and recovery [21]. Applications to hydrophilic and/or charged polymers are very limited. CE is an alternative to LC in this specific, but important, case of complex polyelectrolytes. The molecular reasons behind critical conditions in LC and CE are completely different and are not widely accepted in any case. The electrophoretic mobility always depends on the charge to friction ratio. It does not depend on the ratio of the charge to the size in the case of polyelectrolytes, since the friction is not only hydrodynamic in this case. The critical conditions do not correspond to the free draining model as proposed by Flory, in which the solvent penetrates the polymer chains freely [52]. Electrostatic friction, however, screens the hydrodynamic friction $[53,54]$ and leads to the electrophoretic mobility having a very weak dependence on molar mass for a degree of polymerization generally above $15-20[44,55,56]$. Thus, CE leads to migration independent from molar mass for polyelectrolytes and this corresponds to the critical conditions sought in LC-CC. CE has been used in the critical conditions outside of our group in the separation of pectins [57] and carboxymethylcellulose [58] according to their composition. In our group using CE in the critical conditions (CE-CC) has allowed the investigation of the composition of natural polymers as well as of synthetic polymers. Further, we have also been able to look at the degree of branching of synthetic polymers.

\subsection{Separation by Composition}

3.2.1. Pectin and Carboxymethylcellulose (CMC). CE-CC effectively separates the polysaccharide pectin by composition. Several studies reported the separation of pectin by its degree of substitution (DS, which may include either esterification or methyl-esterification) [57, 59-62]. Within one sample, pectins macromolecules with different degrees of esterification (DE) could be separated. It was later hypothesized that the shape of the peaks could additionally be used to indicate a distribution of methyl esters of pectin within samples [60]. Guillotin et al. [62] established a protocol in which pectin's degree of amidification, degree of methylesterification, and subsequently the degree of substitution could be determined.

Other research involved the use of capillary electrophoresis to determine the DS of carboxymethylcellulose [58]. The study showed the possibility of not only determining the average DS but also determining the heterogeneity/distribution of the compositions of CMC.

3.2.2. Gellan Gum. Gellan gum is a natural polymer which is widely distributed in the environment. Due to its rheological properties it is viewed as a possible stabilizing agent in various industries [63]. Gellan gum's monomer unit structure contains D-glucuronic acid, D-glucose either with or without acyl substituents, and L-rhamnose. The proportion of acyl chains attached to the glucose and the distribution of these

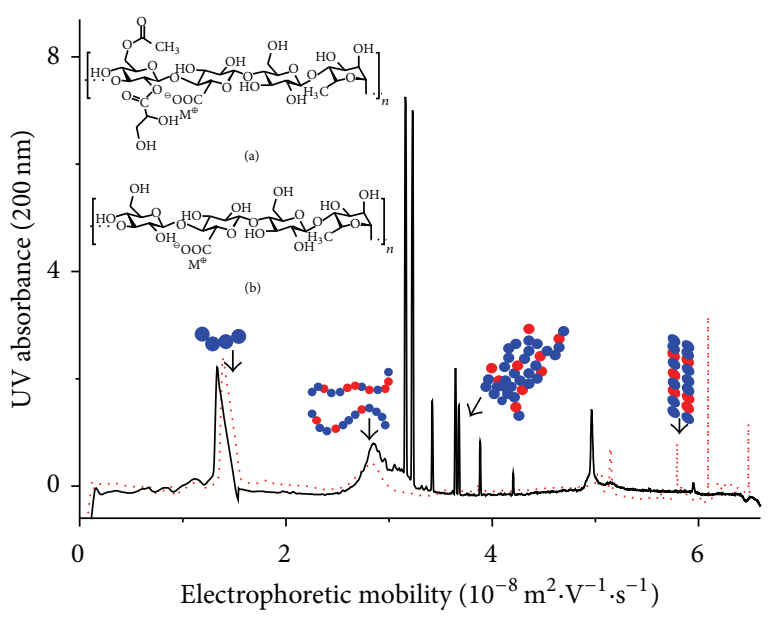

FIGURE 4: Electropherograms of a low acyl gellan gum (dotted red line) and a high-acyl gellan gum (solid black line) (in potassium borate, $\mathrm{pH}$ 9.2), after a few hours of dissolution [39].

acyl chains along the polysaccharide vary from sample to sample. Gellan gum is often characterized by its degree of acylation, which affects its desired properties. CE allowed some separation of gellan gum oligomers according to their degree of polymerization and separation of polymers by their degree of acylation (composition) [39]. CE gave a unique separation of gellan gums that could not be attained with any other existing separation methods. CE could characterize not only a low acyl gellan gum but also a high acyl gellan gum (Figure 4). The latter sample was a turbid dispersion: while obtaining a true solution was not possible, the characterization of this dispersion is relevant for its applications such as the stabilization of carbon nanotubes [64]. Characterization of the high acyl gellan gum showed the presence of aggregates forming during the dissolution of the gellan gum samples and appearing as very narrow peaks due to their very low diffusion coefficients. This illustrates the robustness of the method as it did not require sample filtration (while the background electrolyte still requires filtration). Filtration would have changed the nature of this colloidal sample and should thus not be performed for a meaningful characterization. Complementing the CE separation with a simple pressure mobilization analysis (a qualitative version of Taylor dispersion analysis [65]; see 3.4), the presence of oligomers of gellan gum was confirmed while they had never been identified previously in these gellan gums. Through the CE separation, the oligomers could be separated and quantified. Separation and characterization of this dispersion could only be obtained by $\mathrm{CE}$ or field flow fractionation (FFF). In the most common form of FFF, flow FFF, the oligomers would have been lost through the membrane. The low acyl samples contained more oligomers than the high acyl samples suggesting the occurrence of some degradation during the deacylation process. The electrophoretic mobility is also sensitive to the conformation of gellan gum and complementary to light scattering characterization. A high mobility peak, present in the high acyl sample and becoming more intense in the presence of potassium borate ions, suggests the possibility 


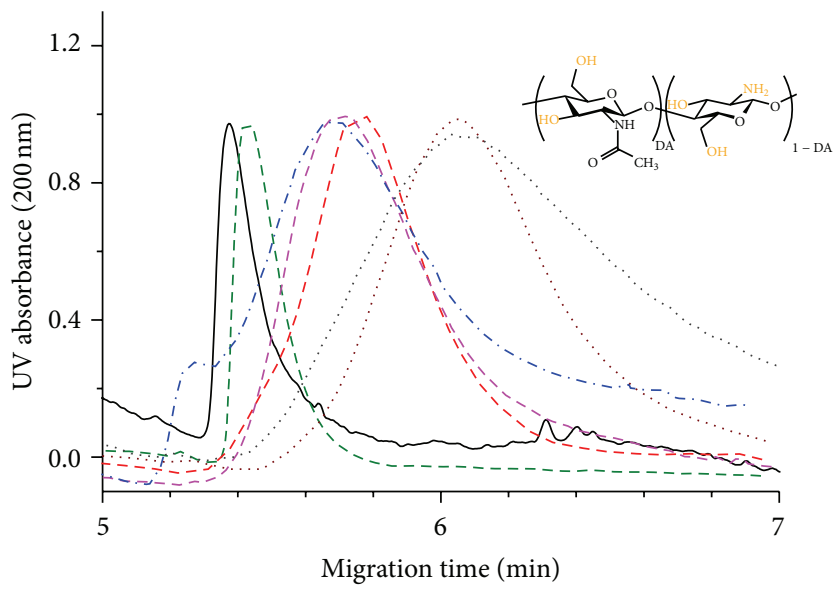

(a)

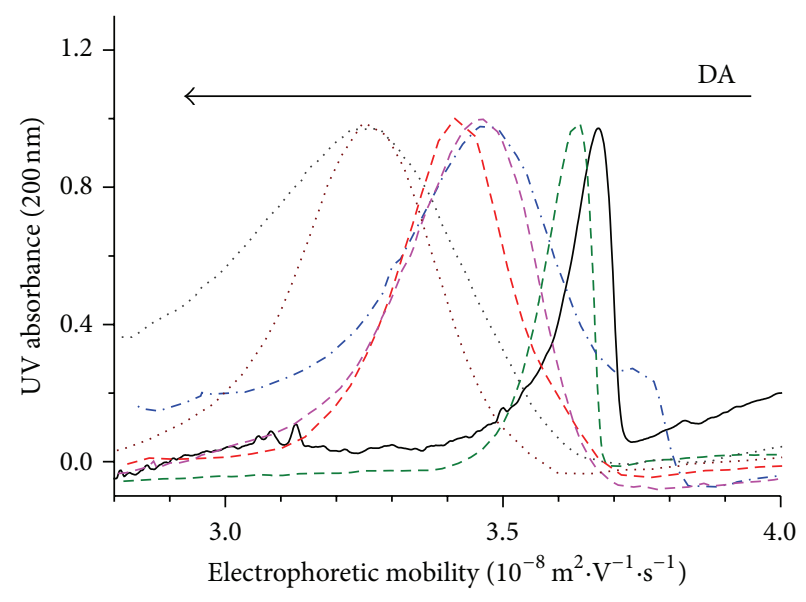

(b)

FIGURE 5: Separation of chitosan samples by their DA with CE (sodium phosphate, $\mathrm{pH}$ 3): electropherogram shown as a function of (a) migration time and (b) apparent electrophoretic mobility. Samples with different degrees of acetylation (weight-average DA determined by NMR spectroscopy) are shown: black solid line (4\%), green dashed line (4.3\%), blue dashed-dotted line (16.5\%), red dashed line (18.7\%), pink dashed line (19.8\%), brown dotted line (22.4\%), and black dotted line (23.6\%) [40].

of a double-helix conformation. This peak differs in mobility and, thus, also suggests that the rest of the macromolecules are in a random coil conformation.

In order of increasing mobility, the electropherograms show the presence of gellan gum oligomers, then gellan gum polymer chains containing different degrees of acylation (random coil conformation), then aggregates of gellan gum chains, and finally gellan gum polymer chains in a helix conformation. The gellan gums studied in this work are copolymers containing both repeating units (not necessarily forming blocks). The top molecular structure represents fully acetylated gellan gum and the bottom one represents fully deacylated gellan gum. The monomer unit is constituted from left to right of one D-glucose with two acyl substituents (one acetyl and one glycerate bearing a diol or glycol), one Dglucuronic acid, one D-glucose without substituents, and one L-rhamnose.

The study undertaken on gellan gum is an example of the robustness of the $\mathrm{CE}$ technique. Whilst allowing the successful separation of complex samples by composition, it also provides information on the conformation of the polymer chains and the presence of aggregates.

3.2.3. Chitosan. Chitosan is a polysaccharide produced from the $\mathrm{N}$-deacetylation of chitin. Chitin is the main component of the shells of crabs and shrimps and can also be found in the cell wall of fungi. It is a renewable resource that it is a large waste product. Chitosan's structure contains $\mathrm{N}$ acetyl-D-glucosamine as well as D-glucosamine units. The composition of this copolymer is quantified by the degree of acetylation (DA), which is the fraction of $N$-acetyl-Dglucosamine. Chitosan is receiving extensive research interest due to its inherent properties. It is biocompatible, antimicrobial, antifungal, biodegradable, and $\mathrm{pH}$-responsive $[66,67]$. However, one limitation of chitosan is in the incomplete characterization by current methods; while being a natural product, there is a large variation among samples. Chitosan is often characterized by its average degree of acetylation (DA) [40]. Chitosan samples are, however, not composed of polymer chains with all the same DA, but they contain a distribution of DAs. The complexity and importance of the distribution of DAs have been revealed recently through a coupling of SEC with ${ }^{1} \mathrm{H}$ NMR spectroscopy [68]; however, it still has not been measured. ${ }^{1} \mathrm{H}$ NMR spectroscopy can determine number-average as well as weight-average DAs [40]. The measurements are accurate, but precise results are time-consuming and alternative methods are often considered. Chitosan is often characterized by only one of its average DAs, which is implicitly and incorrectly assuming the sample is homogeneous in terms of DA, that is, does not have a distribution of DAs.

CE-CC separates chitosan by its degree of acetylation (Figure 5) [40]. Chitosan macromolecules with a lower DA have a higher mobility (at low $\mathrm{pH}$ below the glucosamine monomer unit $\mathrm{pKa}$ ) since they have a higher number of free amino groups which increase their charge and therefore their electrophoretic mobility. Another important attribute revealed in the CE separation is the broadness and shape of the peaks of the chitosan samples. The broadness corresponds to the distribution of DAs and some samples have broader distributions than others (Figure 5). These differences in distributions will likely affect functional properties such as adhesion, biodegradability, and bacteriostaticity [68]. With proper calibration, the CE separation will allow the determination of the distribution of DAs. The distribution can be calculated from the UV signal taking into account the nonlinear relation between electrophoretic mobility and migration, as it is done to calculate molar mass distributions in SEC [10].

We have also used CE to assist in the grafting of synthetic polymers, poly(sodium styrene sulfonate) and poly(methyl methacrylate-co-acrylonitrile), onto a chitosan backbone [41] 


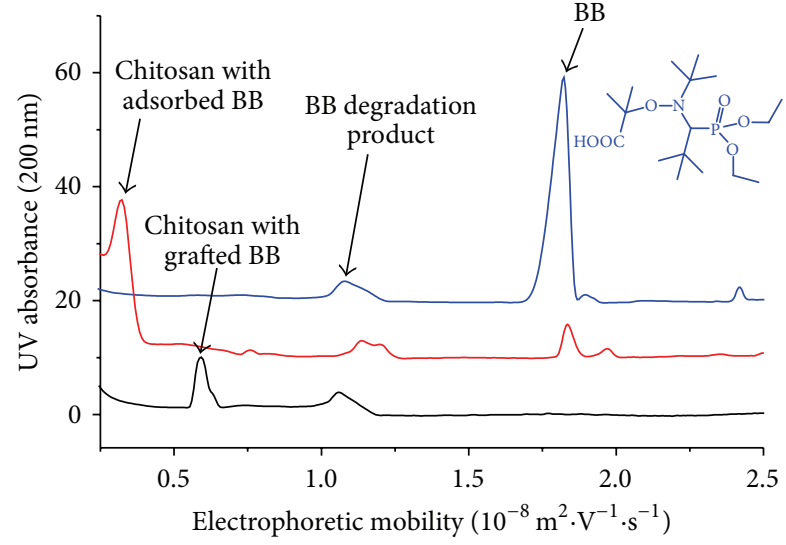

FIgure 6: $\mathrm{CE}$ of pure BlocBuilder (BB, blue line), chitosan with adsorbed BB (red line), and chitosan with grafted BB (red line) in sodium borate buffer ( $\mathrm{pH}$ 9.2) [41].

to address the variability of the mechanical properties of chitosan films [69]. CE could analyze samples produced along the synthetic pathway. This allowed the validation of the grafting process. Chitosan was first functionalized with the introduction of an acrylamide and/or acrylate function. This was followed by a radical addition of BlocBuilder (BB) alkoxyamine to allow a controlled (nitroxide-mediated) polymerization [70] of the grafted monomers. The limited solubility of chitosan, added to the hydrophobicity of the grafted compound, made the complete solubilization of the samples impossible. Despite incomplete dissolution, the robustness of $\mathrm{CE}$ allowed us to analyze these samples: chitosan functionalized with BlocBuilder was separated from pure BlocBuilder (Figure 6). This experiment was undertaken in a high $\mathrm{pH}$ buffer and therefore the negative charge expressed by $\mathrm{BB}$ results in a nonzero mobility in the CE electropherogram whilst the neutral chitosan has no mobility. Pure BB, chitosan covalently functionalized with $\mathrm{BB}$, and chitosan physically mixed with $\mathrm{BB}$ (control) were then injected at the same $\mathrm{pH}$. The chitosan grafted with $\mathrm{BB}$ encounters more hydrodynamic friction than $\mathrm{BB}$ alone which reduces the electrophoretic mobility of the chitosan grafted with $\mathrm{BB}$ in comparison to the pure $\mathrm{BB}$. The method even allowed the discrimination of covalently grafted $\mathrm{BB}$ from $\mathrm{BB}$ adsorbed to chitosan since the latter has a lower electrophoretic mobility in comparison to the grafted sample. This type of separation, neutral polymer chain from slightly modified polymer chain, was also used by the group of Schoenmakers with nonaqueous CE to prove the presence of charged end-groups in part of their poly(2oxazoline) samples [50].

Using both CE and CE-CC a more thorough analysis can be completed on both pure and modified chitosan samples. There is also a possibility of extending the research on chitosan with CE in terms of the determination of the distribution of the degrees of acetylation which has not been previously examined [40].

3.3. Separation by Topology (Branching). There is a large interest in the characterization of water-soluble polyacrylates as their use has increased to include a range of applications from industrial protective coatings to food packaging. The poly(sodium acrylate)s, $\mathrm{PNaA}$, studied by our group were produced by nitroxide-mediated polymerisation (NMP) [70]. The aim of the study was to characterize the branching in PAA using CE-CC. Different topologies of the PAA samples, linear, hyperbranched, and three-arm star, were separated within $15 \mathrm{~min}$ [42]. Figure 7 presents the $\mathrm{CE}$ results both as raw data, as a function of migration time and as EOF corrected data, as a function of electrophoretic mobility. This highlights the importance of converting the results to electrophoretic mobility plots as a trend is not seen in the migration time results due to the variation in $\mathrm{EOF}$ between injections. When the EOF correction is made, it can be seen that the hyperbranched polymer exhibits the lowest electrophoretic mobility followed by three-arm star and finally the linear ones. The differences in electrophoretic mobility can be explained by a decrease in the effective charge of the branched samples when compared to the linear samples. The results obtained were highly repeatable and reproducible with relative standard deviations (RSD) values below $1.6 \%$. The separations were also successfully reproduced in a different buffer and whilst they produced different electrophoretic mobility values (as expected, due to the different counterions), the electrophoretic mobility remained lower for the more branched structures. This study highlights also the accuracy (related to the reproducibility of the separation) of the technique.

The CE results obtained also provided information regarding the homogeneity of the branching topology. These samples are expected to have a controlled molar mass owing to the reversible termination with the nitroxide but the broadness of the peaks obtained suggests some heterogeneity in the branching structure. The broad range of electrophoretic mobilities is attributed to a broad range of branching topologies produced in the polymerization.

The CE-CC separation was also shown to be influenced by end-groups. In Figure 8 the red solid line represents PAA with a SG1 [N-tert-butyl-N-(1-diethylphosphono-2,2dimethylpropyl) nitroxide] moiety as the end-group. There is a marked difference between this sample and the sample with the PAA that has been heated in the presence of thiophenol to replace the SG1 end-groups with hydrogen. The electrophoretic mobility increases with the removal of the SG1 end-group, as expected, since the bulky SG1 molecule would contribute to the hydrodynamic friction of the PAA chains more than hydrogen (and neither contributes to its charge). The result also suggests heterogeneity of the sample in terms of branching. The thiophenol treatment was also applied to a PAA sample obtained from the hydrolysis of poly(t-butyl acrylate) and CE-CC showed that the hydrolysis of poly(t-butyl acrylate) did not just cleave the targeted $t$ butyl groups, but also likely lead to some degradation of the SG1 end-group. This led to a greater heterogeneity of the sample and is expressed in the broadness of the peak.

Through CE-CC, we were able to separate polymers by their topology (branching) as well as by their end-groups. The ability of CE-CC to separate based on the presence of SG1 (control agent used for nitroxide-mediated polymerization) 


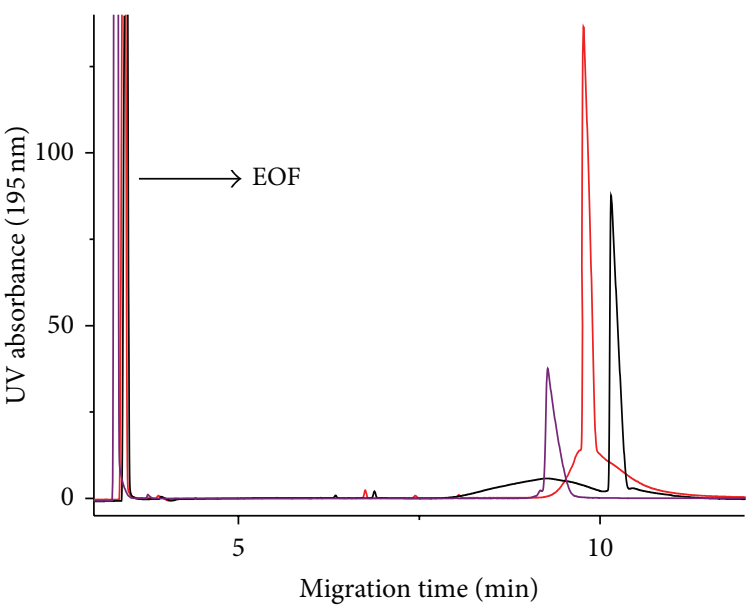

(a)

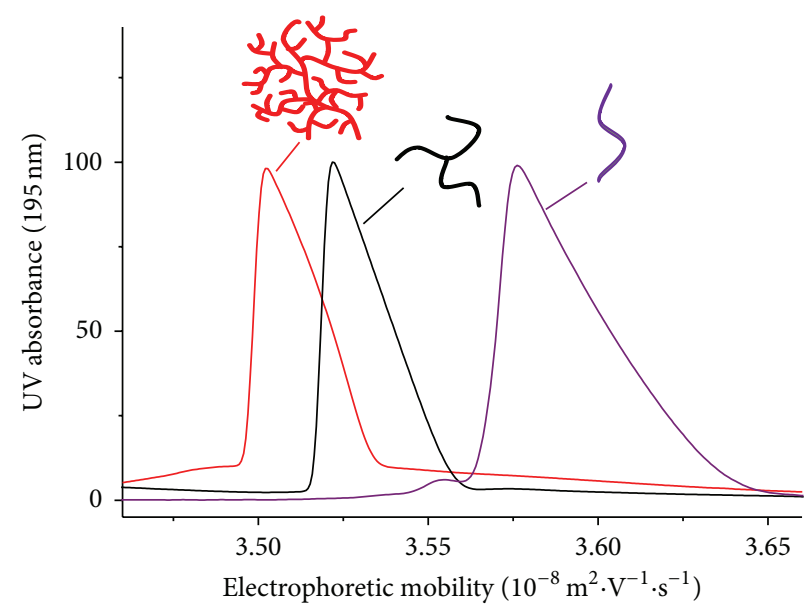

(b)

FIGURE 7: Separation of linear (purple line), three-arm star (black line), and hyperbranched (red line) poly(sodium acrylate) by capillary electrophoresis in sodium borate buffer ( $\mathrm{pH}$ 9.2) shown as a function of (a) migration time and (b) electrophoretic mobility, which is a more reproducible quantity than the former [42].

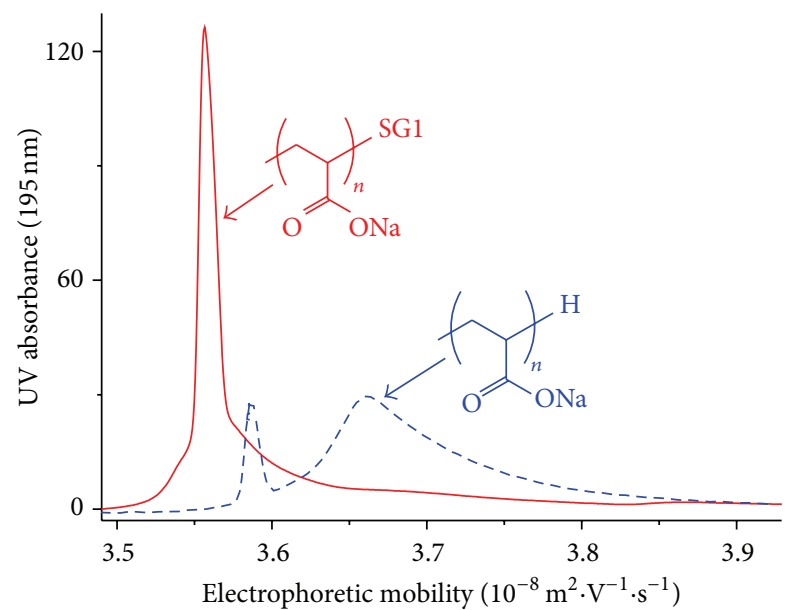

FIGURE 8: Electrophoretic mobility distributions of a PNaA obtained by nitroxide-mediated polymerization of acrylic acid initiated by the monofunctional initiator Monams (red solid line) followed by cleavage of the SG1 end-group by treatment with thiophenol (blue dashed line) in sodium borate ( $\mathrm{pH}$ 9.2) [42].

reveals information regarding the "livingness" of the obtained sample (allowing continuing reacting through NMP) [42, 71]. This allows the optimization of the method used to produce the sample and provides information regarding further functionalization of the PAA.

3.4. Size Determination with TDA. One limitation of CE for polymer characterization, compared to multiple detection SEC [16], is the limited number of detectors, especially the lack of molar mass sensitive detectors. The group of Cottet is, however, bridging this gap rapidly by demonstrating that the CE separation can be coupled to Taylor dispersion analysis (TDA). TDA is a method that does not involve separation but allows the determination of the diffusion coefficient/hydrodynamic radius of a sample. TDA has been looked at previously to obtain diffusion coefficients in liquid systems [72, 73]. Further it has proven to be practical in the size characterization of macromolecules and particles of virtually any molar mass $[65,74]$. TDA has several advantages including that it is an absolute method, meaning that no calibration is required. The group of Cottet has shown that a CE instrument is particularly well suited to carry out TDA [65, 75]. Le Saux and Cottet [76] coupled $\mathrm{CE}$ and TDA. A copolymer mixture of 2-acrylamido-2methylpropanesulfonate/acrylamide and DNA was injected into a fused silica capillary. The mixture was separated in $\mathrm{CE}$ conditions (with an electric field) and then pressure was used to push the samples to the detection window where the analysis took place. The experiment proved that the coupling of CE and TDA allowed not only a complete separation of the copolymer from the DNA in the mixture, but also the successful determination of the diffusion coefficient of both the copolymer and the DNA. A successful coupling of CE with TDA allowed a combination of a high performance and throughput method with an absolute method for the calculation of diffusion coefficients [77]. The diffusion coefficient can then be related to the hydrodynamic volume of the macromolecule as it is classically done in light scattering by the following equation:

$$
D=k T 6 \pi \eta r
$$

where $k$ is the Boltzmann constant, $T$ is the temperature, $\eta$ is the viscosity of the solvent, and $r$ is the hydrodynamic radius of the macromolecule [78].

The relation of the hydrodynamic radius to the molar mass is complex and is influenced by branching and copolymer composition as discussed in Section 1 for SEC [16]. 


\section{Conclusions and Future Directions}

Characterization by capillary electrophoresis involves both separation and characterization of complex polymers. This review outlined the broad range of samples that can be analyzed with CE. The characterization of complex polymers is significant for various industries including food, biomedical, energy/fuel, and materials (such as paint and bioplastics) industries. The robustness of the method, especially the minimal sample preparation, is one of the main strengths of the method (shared with field flow fractionation). The continual development of the methods in CE and their coupling with other techniques such as TDA widens the scope and depth of the possible characterization and meets the ever-growing needs of progressively increasing complex macromolecular structures for increasingly advanced applications. CE in the critical conditions (CE-CC) has the most potential and can be applied to a wide variety of charged polymers to characterize their topology, composition, or end-groups. The method is complementary to SEC.

Future directions will look into further characterizing complex polyelectrolytes, for example, in terms of determination of the molar mass distribution of one block in block copolymers, or in terms of sugars quantification in food samples. The different distributions related to branching will also be studied. CE coupled with TDA is a very useful and simple technique that will definitely be examined further as it is able to provide extremely valuable information regarding the size and shape of sample molecules by the calculation of their diffusion coefficient. Its simplicity and being an absolute method mean that it can be applied to a variety of samples investigated by our research group and other polymer research groups.

\section{Conflict of Interests}

The authors declare that there is no conflict of interests regarding the publication of this paper.

\section{Acknowledgments}

Patrice Castignolles and Marianne Gaborieau would like to thank Professor Herve Cottet (University of Montpellier II) and Professor Emily Hilder (ACROSS, University of Tasmania) for discussions over the last 10 years. The authors thank Alison Maniego, Adam Sutton, James Oliver, Danielle Taylor, and their entire macromolecular characterization team.

\section{References}

[1] A. M. Striegel, J. J. Kirkland, W. W. Yau, and D. D. Bly, Modern Size Exclusion Chromatography, John Wiley \& Sons, Hoboken, NJ, USA, 2009.

[2] R. J. Bruessau, "Experiences with interlaboratory GPC experiments," Macromolecular Symposia, vol. 110, pp. 15-32, 1996.

[3] D. Berek, "Size exclusion chromatography-a blessing and a curse of science and technology of synthetic polymers," Journal of Separation Science, vol. 33, no. 3, pp. 315-335, 2010.
[4] H. G. Barth and F. J. Carlin Jr., "A review of polymer shear degradation in size-exclusion chromatography," Journal of Liquid Chromatography, vol. 7, no. 9, pp. 1717-1738, 1984.

[5] E. Uliyanchenko, S. van der Wal, and P. J. Schoenmakers, "Deformation and degradation of polymers in ultra-highpressure liquid chromatography," Journal of Chromatography A, vol. 1218, no. 39, pp. 6930-6942, 2011.

[6] L. K. Kostanski, D. M. Keller, and A. E. Hamielec, "Sizeexclusion chromatography-A review of calibration methodologies," Journal of Biochemical and Biophysical Methods, vol. 58, no. 2, pp. 159-186, 2004.

[7] M. Netopilík and P. Kratochvíl, "Polystyrene-equivalent molecular weight versus true molecular weight in size-exclusion chromatography," Polymer, vol. 44, no. 12, pp. 3431-3436, 2003.

[8] Y. Guillaneuf and P. Castignolles, "Using apparent molecular weight from SEC in controlled/living polymerization and kinetics of polymerization," Journal of Polymer Science A: Polymer Chemistry, vol. 46, no. 3, pp. 897-911, 2008.

[9] A. E. Hamielec and A. C. Ouano, "Generalized universal molecular-weight calibration parameter in GPC," Journal of Liquid Chromatography, vol. 1, pp. 111-120, 1978.

[10] M. Gaborieau, R. G. Gilbert, A. Gray-Weale, J. M. Hernandez, and P. Castignolles, "Theory of multiple-detection size-exclusion chromatography of complex branched polymers," Macromolecular Theory and Simulations, vol. 16, no. 1, pp. 13-28, 2007.

[11] M. Gaborieau, J. Nicolas, M. Save et al., "Separation of complex branched polymers by size-exclusion chromatography probed with multiple detection," Journal of Chromatography A, vol. 1190, no. 1-2, pp. 215-223, 2008.

[12] L. Couvreur, G. Piteau, P. Castignolles et al., "Pulsed-laser radical polymerization and propagation kinetic parameters of some alkyl acrylates," Macromolecular Symposia, vol. 174, pp. 197-207, 2001.

[13] P. Castignolles, R. Graf, M. Parkinson, M. Wilhelm, and M. Gaborieau, "Detection and quantification of branching in polyacrylates by size-exclusion chromatography (SEC) and meltstate 13C NMR spectroscopy," Polymer, vol. 50, no. 11, pp. 23732383, 2009.

[14] T. Junkers, M. Schneider-Baumann, S. S. P. Koo, P. Castignolles, and C. Barner-Kowollik, "Determination of propagation rate coefficients for methyl and 2-ethylhexyl acrylate via high frequency PLP-SEC under consideration of the impact of chain branching," Macromolecules, vol. 43, no. 24, pp. 10427-10434, 2010.

[15] P. Castignolles, "Transfer to polymer and long-chain branching in PLP-SEC of acrylates," Macromolecular Rapid Communications, vol. 30, no. 23, pp. 1995-2001, 2009.

[16] M. Gaborieau and P. Castignolles, "Size-exclusion chromatography (SEC) of branched polymers and polysaccharides," Analytical and Bioanalytical Chemistry, vol. 399, no. 4, pp. 14131423, 2011.

[17] E. Uliyanchenko, P. J. C. H. Cools, S. van der Wal, and P. J. Schoenmakers, "Comprehensive two-dimensional ultrahighpressure liquid chromatography for separations of polymers," Analytical Chemistry, vol. 84, no. 18, pp. 7802-7809, 2012.

[18] M. Rollet, D. Glé, T. N. T. Phan, Y. Guillaneuf, D. Bertin, and D. Gigmes, "Characterization of functional poly(ethylene oxide)s and their corresponding polystyrene block copolymers by liquid chromatography under critical conditions in organic solvents," Macromolecules, vol. 45, no. 17, pp. 7171-7178, 2012.

[19] M. Al Samman, W. Radke, A. Khalyavina, and A. Lederer, "Retention behavior of linear, branched, and hyperbranched 
polyesters in interaction liquid chromatography," Macromolecules, vol. 43, no. 7, pp. 3215-3220, 2010.

[20] W. Lee, D. Cho, T. Chang, K. J. Hanley, and T. P. Lodge, "Characterization of polystyrene-b-polyisoprene diblock copolymers by liquid chromatography at the chromatographic critical condition," Macromolecules, vol. 34, no. 7, pp. 2353-2358, 2001.

[21] A. Favier, C. Petit, E. Beaudoin, and D. Bertin, "Liquid chromatography at the critical adsorption point (LC-CAP) of high molecular weight polystyrene: pushing back the limits of reduced sample recovery," E-Polymers, pp. 1-15, 2009.

[22] E. Beaudoin, A. Favier, C. Galindo et al., "Reduced sample recovery in liquid chromatography at critical adsorption point of high molar mass polystyrene," European Polymer Journal, vol. 44, no. 2, pp. 514-522, 2008.

[23] R. Weinberger, Practical Capillary Electrophoresis, CA Academic Press, San Diego, Calif, USA, 2000.

[24] H. Cottet and P. Gareil, "Separation of synthetic (co)polymers by capillary electrophoresis techniques.," Methods in Molecular Biology, vol. 384, pp. 541-567, 2008.

[25] H. Miramon, F. Cavelier, J. Martinez, and H. Cottett, "Highly resolutive separations of hardly soluble synthetic polypeptides by capillary electrophoresis," Analytical Chemistry, vol. 82, no. 1, pp. 394-399, 2010.

[26] W. Vayaboury, O. Giani, H. Cottet, S. Bonaric, and F. Schué, "Mechanistic Study Of $\alpha$-amino acid N-carboxyanhydride (NCA) polymerization by capillary electrophoresis," Macromolecular Chemistry and Physics, vol. 209, no. 15, pp. 1628-1637, 2008.

[27] M. R. Aguilar, A. Gallardo, J. San Román, and A. Cifuentes, "Micellar electrokinetic chromatography: a powerful analytical tool to study copolymerization reactions involving ionic species," Macromolecules, vol. 35, no. 22, pp. 8315-8322, 2002.

[28] I. Spiridon and V. I. Popa, "Hemicelluloses: major sources, properties and applications," in Monomers, Polymers and Composites from Renewable Resources, M. N. Belgacem and A. Gandini, Eds., chapter 13, Elsevier, Amsterdam, The Netherlands, 2008.

[29] S. Brudin and P. Schoenmakers, "Analytical methodology for sulfonated lignins," Journal of Separation Science, vol. 33, no. 3, pp. 439-452, 2010.

[30] J. D. Oliver, M. Gaborieau, E. F. Hilder, and P. Castignolles, "Simple and robust determination of monosaccharides in plant fibers in complex mixtures by capillary electrophoresis and high performance liquid chromatography," Journal of Chromatography A, vol. 1291, pp. 179-186, 2013.

[31] M. Verzele, G. Simoens, and F. van Damme, "A critical review of some liquid chromatography systems for the separation of sugars," Chromatographia, vol. 23, no. 4, pp. 292-300, 1987.

[32] S. Rovio, H. Simolin, K. Koljonen, and H. Sirén, "Determination of monosaccharide composition in plant fiber materials by capillary zone electrophoresis," Journal of Chromatography A, vol. 1185, no. 1, pp. 139-144, 2008.

[33] S. Rovio, J. Yli-Kauhaluoma, and H. Sirén, "Determination of neutral carbohydrates by CZE with direct UV detection," Electrophoresis, vol. 28, no. 17, pp. 3129-3135, 2007.

[34] A. E. Vorndran, P. J. Oefner, H. Scherz, and G. K. Bonn, "Indirect UV detection of carbohydrates in capillary zone electrophoresis," Chromatographia, vol. 33, no. 3-4, pp. 163-168, 1992.

[35] J. D. Oliver, M. Gaborieau, and P. Castignolles, "Ethanol determination using pressure mobilization and free solution capillary electrophoresis by photo-oxidation assisted UV detection," Journal of Chromatography A, vol. 1348, pp. 150-157, 2014.
[36] J. D. Oliver, A. T. Sutton, N. Karu et al., "Simple and robust monitoring of ethanol fermentations by capillary electrophoresis," Biotechnology and Applied Biochemistry, 2014.

[37] J. D. Oliver, A. A. Rosser, C. M. Fellows et al., "Understanding and improving direct UV detection of monosaccharides and disaccharides in free solution capillary electrophoresis," Analytica Chimica Acta, vol. 809, pp. 183-193, 2014.

[38] P. Castignolles, M. Gaborieau, E. F. Hilder, E. Sprang, C. J. Ferguson, and R. G. Gilbert, "High-resolution separation of oligo(acrylic acid) by capillary zone electrophoresis," Macromolecular Rapid Communications, vol. 27, no. 1, pp. 42-46, 2006.

[39] D. L. Taylor, C. J. Ferris, A. R. Maniego, P. Castignolles, M. In Het Panhuis, and M. Gaborieau, "Characterization of gellan gum by capillary electrophoresis," Australian Journal of Chemistry, vol. 65, no. 8, pp. 1156-1164, 2012.

[40] M. Mnatsakanyan, J. J. Thevarajah, R. S. Roi, A. Lauto, M. Gaborieau, and P. Castignolles, "Separation of chitosan by degree of acetylation using simple free solution capillary electrophoresis," Analytical and Bioanalytical Chemistry, vol. 405, no. 21, pp. 6873-6877, 2013.

[41] C. Lefay, Y. Guillaneuf, G. Moreira et al., "Heterogeneous modification of chitosan via nitroxide-mediated polymerization," Polymer Chemistry, vol. 4, no. 2, pp. 322-328, 2013.

[42] A. R. Maniego, D. Ang, Y. Guillaneuf et al., "Separation of poly(acrylic acid) salts according to topology using capillary electrophoresis in the critical conditions," Analytical and Bioanalytical Chemistry, vol. 405, no. 28, pp. 9009-9020, 2013.

[43] C. Sarazin, N. Delaunay, C. Costanza, V. Eudes, J. Mallet, and P. Gareil, "New avenue for mid-UV-range detection of underivatized carbohydrates and amino acids in capillary electrophoresis," Analytical Chemistry, vol. 83, no. 19, pp. 7381-7387, 2011.

[44] H. Cottet and P. Gareil, "From small charged molecules to oligomers: a semiempirical approach to the modeling of actual mobility in free solution," Electrophoresis, vol. 21, pp. 1493-1504, 2000.

[45] M. Siauw, B. S. Hawkett, and S. Perrier, "Short chain amphiphilic diblock co-oligomers via RAFT polymerization," Journal of Polymer Science A: Polymer Chemistry, vol. 50, no. 1, pp. 187198, 2012.

[46] C. J. Ferguson, R. J. Hughes, D. Nguyen et al., "Ab initio emulsion polymerization by RAFT-controlled self-assembly," Macromolecules, vol. 38, no. 6, pp. 2191-2204, 2005.

[47] I. Lacík, M. Stach, P. Kasak et al., "SEC analysis of poly(acrylic acid ) and poly(methacrylic acid)," Macromolecular Chemistry and Physics, 2014.

[48] M. Gaborieau, T. J. Causon, Y. Guillaneuf, E. F. Hilder, and P. Castignolles, "Molecular weight and tacticity of oligoacrylates by capillary electrophoresis-mass spectrometry," Australian Journal of Chemistry, vol. 63, no. 8, pp. 1219-1226, 2010.

[49] C. M. Guttman, K. M. Flynn, W. E. Wallace, and A. J. Kearsley, "Quantitative mass spectrometry and polydisperse materials: creation of an absolute molecular mass distribution polymer standard," Macromolecules, vol. 42, no. 5, pp. 1695-1702, 2009.

[50] A. Chojnacka, K. Kempe, H. C. van de Ven et al., "Molar mass, chemical-composition, and functionality-type distributions of poly(2-oxazoline)s revealed by a variety of separation techniques," Journal of Chromatography A, vol. 1265, pp. 123-132, 2012.

[51] E. B. Fitzgerald and R. M. Fuoss, "Polyelectrolytes .11. electrophoresis in solutions of poly-4-vinyl-N-N-butylpyridinium bromide," Journal of Polymer Science, vol. 14, pp. 329-339, 1954. 
[52] P. J. Flory, "Configurational and frictional properties of the polymer molecule in dilute solution," in Principles of Polymer Chemistry, Cornell University Press, Ithaca, NY, USA, 1st edition, 1953.

[53] M. Muthukumar, "Theory of electrophoretic mobility of a polyelectrolyte in semidilute solutions of neutral polymers," Electrophoresis, vol. 17, no. 6, pp. 1167-1172, 1996.

[54] J. L. Barrat and J. F. Joanny, “Theory of polyelectrolyte solutions," in Advances in Chemical Physics, Vol Xciv, John Wiley \& Sons, New York, NY, USA, 1996.

[55] N. C. Stellwagen, C. Gelfi, and P. G. Righetti, "The Free Solution Mobility of DMA," Biopolymers, vol. 42, no. 6, pp. 687-703, 1997.

[56] H. Cottet, P. Gareil, O. Theodoly, and C. E. Williams, "A semiempirical approach to the modeling of the electrophoretic mobility in free solution: application to polystyrenesulfonates of various sulfonation rates," Electrophoresis, vol. 21, no. 17, pp. 3529-3540, 2000.

[57] H. J. Zhong, M. A. K. Williams, R. D. Keenan, D. M. Goodall, and C. Rolin, "Separation and quantification of pectins using capillary electrophoresis: a preliminary study," Carbohydrate Polymers, vol. 32, no. 1, pp. 27-32, 1997.

[58] K. A. Oudhoff, F. A. Buijtenhuijs, P. H. Wijnen, P. J. Schoenmakers, and W. T. Kok, "Determination of the degree of substitution and its distribution of carboxymethylcelluloses by capillary zone electrophoresis," Carbohydrate Research, vol. 339, no. 11, pp. 1917-1924, 2004.

[59] C.-M. Jiang, M.-C. Wu, W.-H. Chan, and H.-M. Chang, "Determination of random- and blockwise-type de-esterified pectins by capillary zone electrophoresis," Journal of Agricultural and Food Chemistry, vol. 49, no. 11, pp. 5584-5588, 2001.

[60] M. A. K. Williams, T. J. Foster, and H. A. Schols, "Elucidation of pectin methylester distributions by capillary electrophoresis," Journal of Agricultural and Food Chemistry, vol. 51, no. 7, pp. 1777-1781, 2003.

[61] C.-M. Jiang, S.-C. Liu, M.-C. Wu, W.-H. Chang, and H.M. Chang, "Determination of the degree of esterification of alkaline de-esterified pectins by capillary zone electrophoresis," Food Chemistry, vol. 91, no. 3, pp. 551-555, 2005.

[62] S. E. Guillotin, E. J. Bakx, P. Boulenguer, H. A. Schols, and A. G. J. Voragen, "Determination of the degree of substitution, degree of amidation and degree of blockiness of commercial pectins by using capillary electrophoresis," Food Hydrocolloids, vol. 21, no. 3, pp. 444-451, 2007.

[63] C. J. Ferris, K. J. Gilmore, G. G. Wallace, and M. in het Panhuis, "Modified gellan gum hydrogels for tissue engineering applications," Soft Matter, vol. 9, no. 14, pp. 3705-3711, 2013.

[64] C. John Ferrisa and M. in het Panhuism, "Conducting biomaterials based on gellan gum hydrogels," Soft Matter, vol. 5, no. 18 , pp. 3430-3437, 2009.

[65] H. Cottet, J. Biron, and M. Martin, "Taylor dispersion analysis of mixtures," Analytical Chemistry, vol. 79, no. 23, pp. 9066-9073, 2007.

[66] A. Domard, "A perspective on 30 years research on chitin and chitosan," Carbohydrate Polymers, vol. 84, no. 2, pp. 696-703, 2011.

[67] I. Aranaz, M. Mengíbar, R. Harris et al., "Functional characterization of chitin and chitosan," Current Chemical Biology, vol. 3, no. 2, pp. 203-230, 2009.

[68] S. Nguyen, S. Hisiger, M. Jolicoeur, F. M. Winnik, and M. D. Buschmann, "Fractionation and characterization of chitosan by analytical SEC and $1 \mathrm{H}$ NMR after semi-preparative SEC," Carbohydrate Polymers, vol. 75, no. 4, pp. 636-645, 2009.
[69] C. Gartner, B. L. López, L. Sierra, R. Graf, H. W. Spiess, and M. Gaborieau, "Interplay between structure and dynamics in chitosan films investigated with solid-state NMR, dynamic mechanical analysis, and X-ray diffraction," Biomacromolecules, vol. 12, no. 4, pp. 1380-1386, 2011.

[70] J. Nicolas, Y. Guillaneuf, C. Lefay, D. Bertin, D. Gigmes, and B. Charleux, "Nitroxide-mediated polymerization," Progress in Polymer Science, vol. 38, no. 1, pp. 63-235, 2013.

[71] D. Gigmes, D. Bertin, C. Lefay, and Y. Guillaneuf, "Kinetic modeling of nitroxide-mediated polymerization: conditions for living and controlled polymerization," Macromolecular Theory and Simulations, vol. 18, no. 7-8, pp. 402-419, 2009.

[72] A. C. Ouano, "Diffusion in liquid systems. I. A simple and fast method of measuring diffusion constants," Industrial and Engineering Chemistry Fundamentals, vol. 11, no. 2, pp. 268-271, 1972.

[73] K. C. Pratt and W. A. Wakeham, "Mutual diffusion-coefficient of ethanol-water mixtures-determination by a rapid, new method," Proceedings of the Royal Society of London AMathematical Physical and Engineering Sciences, vol. 336, pp. 393-406, 1974.

[74] R. Callendar and D. G. Leaist, "Diffusion coefficients for binary, ternary, and polydisperse solutions from peak-width analysis of Taylor dispersion profiles," Journal of Solution Chemistry, vol. 35, no. 3, pp. 353-379, 2006.

[75] H. Cottet, M. Martin, A. Papillaud, E. Souaïd, H. Collet, and A. Commeyras, "Determination of dendrigraft poly-L-lysine diffusion coefficients by Taylor dispersion analysis," Biomacromolecules, vol. 8, no. 10, pp. 3235-3243, 2007.

[76] T. le Saux and H. Cottet, "Size-based characterization by the coupling of capillary electrophoresis to taylor dispersion analysis," Analytical Chemistry, vol. 80, no. 5, pp. 1829-1832, 2008.

[77] A. Ibrahim, R. Meyrueix, G. Pouliquen, Y. P. Chan, and H. Cottet, "Size and charge characterization of polymeric drug delivery systems by Taylor dispersion analysis and capillary electrophoresis," Analytical and Bioanalytical Chemistry, vol. 405, no. 16, pp. 5369-5379, 2013.

[78] J. R. Lakowicz, Principles of Fluorescence Spectroscopy, Springer, New York, NY, USA, 2007. 

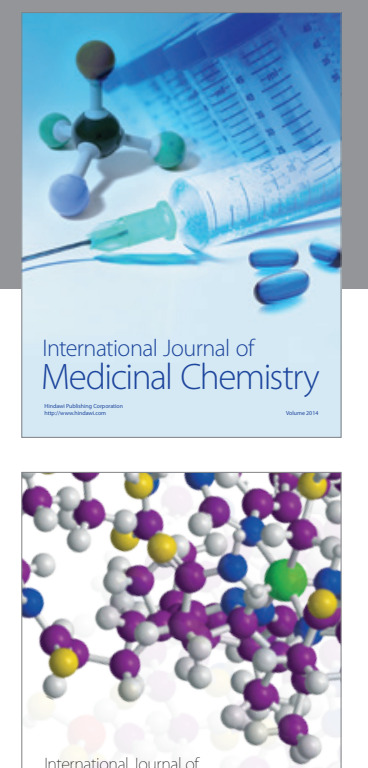

\section{Carbohydrate} Chemistry

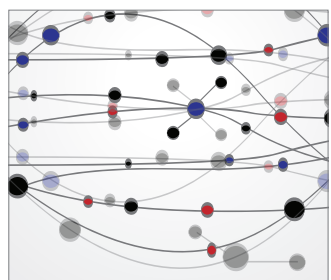

The Scientific World Journal
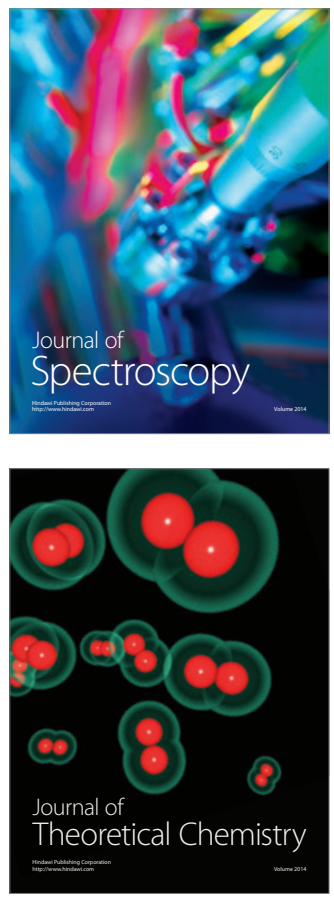
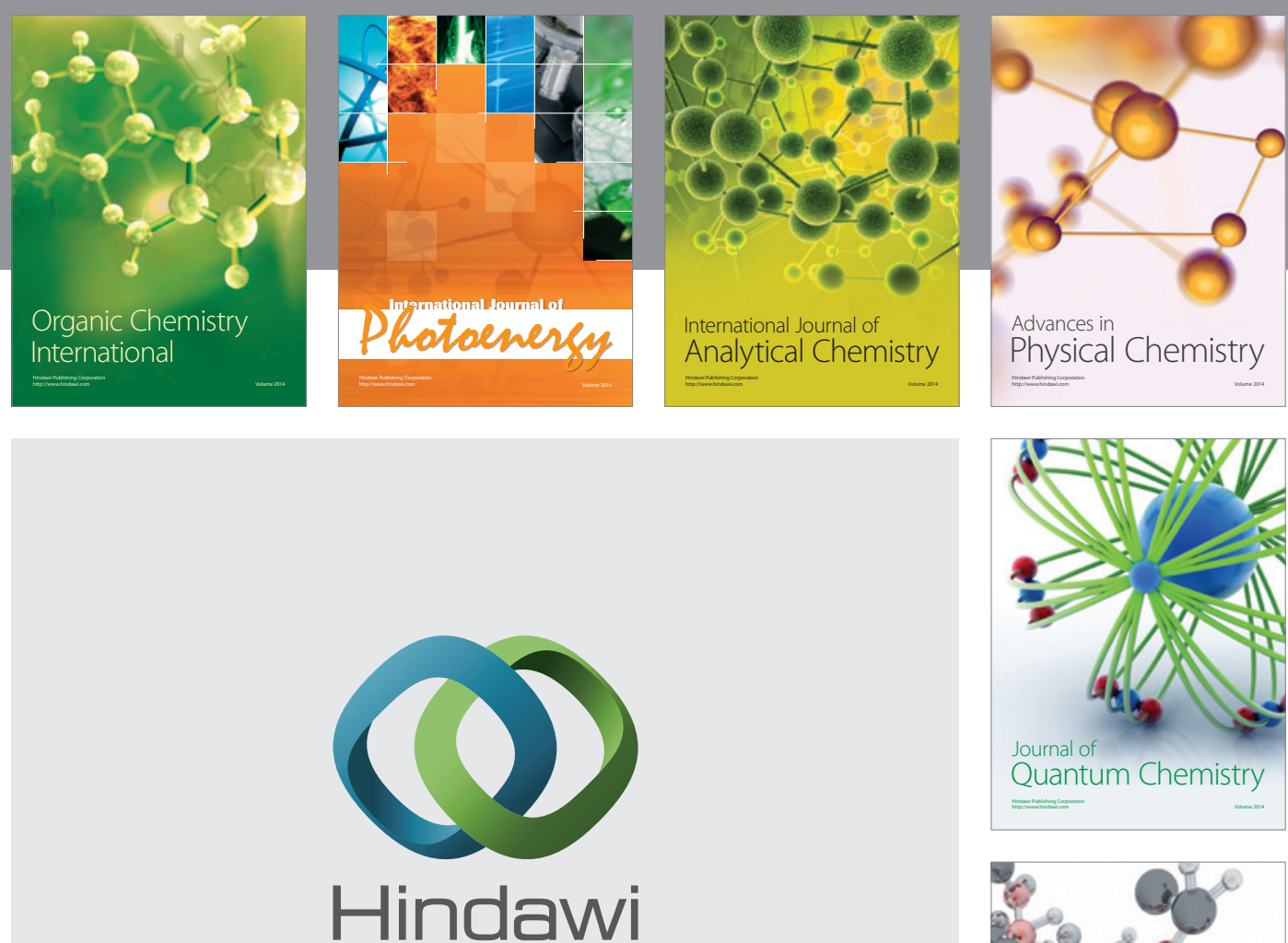

Submit your manuscripts at

http://www.hindawi.com

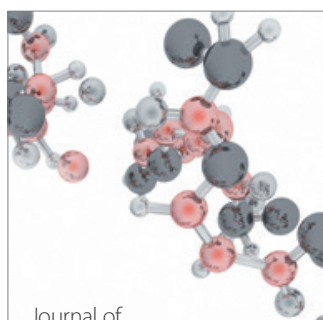

Analytical Methods

in Chemistry

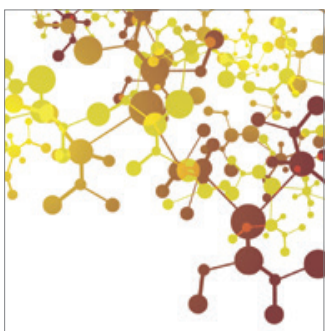

Journal of

Applied Chemistry

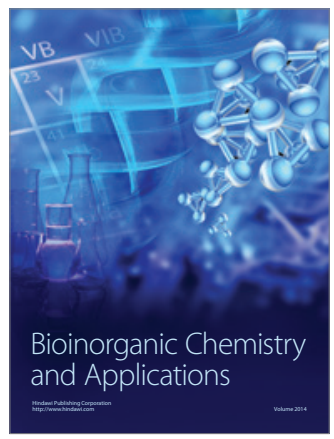

Inorganic Chemistry
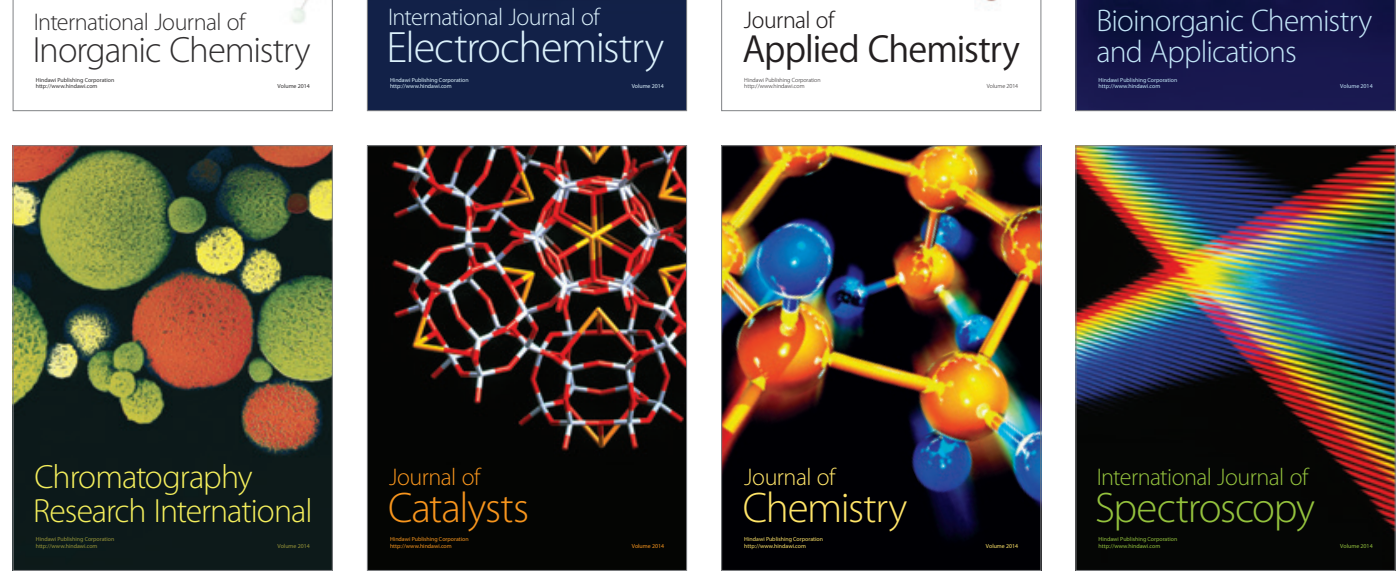